\title{
Reliability and validity of the Persian version of the spinal cord injury lifestyle scale and the health behavior questionnaire in persons with spinal cord injury
}

\author{
Maryam Shabany ${ }^{1} \cdot$ Alireza Nikbakht Nasrabadi $^{1,2} \cdot$ Vafa Rahimi-Movaghar $^{3} \cdot$ Mohammad Ali Mansournia $^{4}$. \\ Nooredin Mohammadi ${ }^{5}$ Sheri D. Pruitt ${ }^{6}$
}

Received: 14 September 2017 / Revised: 29 November 2017 / Accepted: 16 December 2017 / Published online: 16 January 2018

(c) International Spinal Cord Society 2018

\begin{abstract}
Study design Cross-sectional psychometric study.

Objective To evaluate the reliability and validity of a spinal cord injury lifestyle scale (SCILS) and Health Behavior Questionnaire (HBQ) in the Persian language for persons with spinal cord injury (SCI).

Setting Participants were selected among those referred to health centers and the Brain and Spinal Cord Injury Research Center.

Method In accordance with standard procedure for translation, two questionnaires, the SCILS and HBQ, were translated using a forward and backward translation approach by professional translators. Face validity of the questionnaires was assessed by ten persons with SCI and content validity was agreed upon by 12 professors from health care teaching universities. To test the final versions of both questionnaires, 97 persons with SCI were included using a consecutive sampling method. Other questionnaires were used to assess concurrent validity (secondary impairment checklist, as well as SCILS and HBQ) and convergent validity (impact of event scale revised, brief symptom inventory, beck depression inventory, and functional independence measure).

Results Internal consistency of SCILS and HBQ, assessed by Cronbach's alpha, was 0.75 for SCILS and 0.85 for HBQ. Test-retest reliability intraclass correlations were 0.86 and 0.92 for SCILS and HBQ, respectively. The number of current secondary impairments had a significant and negative correlation with SCILS $(r=-0.22, P<0.001)$, but it was not correlated with HBQ. SCILS had a significant and strong correlation with HBQ $(r=0.65, P<0.001)$.
\end{abstract}

Conclusion SCILS and HBQ can be used for measuring the health behavior of persons with SCI in Iran.

Maryam Shabany and Alireza Nikbakht Nasrabadi are co-first authors.

Electronic supplementary material The online version of this article (https://doi.org/10.1038/s41393-017-0056-9) contains supplementary material, which is available to authorized users.

Mohammad Ali Mansournia mansournia_ma@yahoo.com

1 Brain and Spinal Cord Injury Research Center, Neuroscience Institute, Tehran University of Medical Sciences, Tehran, Iran

2 Dept. of Medical Surgical Nursing \& Deputy Dean for International Affairs, Nursing and Midwifery School, Tehran University of Medical Sciences, Tehran, Iran

3 Sina Trauma and Surgery Research Center, Tehran University of

\section{Introduction}

A World Health Organization (WHO) report regarding health behaviors in 35 countries showed that about $60 \%$ of one's quality of life and health status depend on lifestyle and personal behaviors [1]. Assessment of health behaviors in individuals with chronic conditions is necessary in order to improve or prevent undesired physical and mental

Medical Sciences, Tehran, Islamic Republic of Iran

4 Department of Epidemiology and Biostatistics, School of Public Health, Tehran University of Medical Sciences, Tehran, Iran

5 Dept. of Critical Care Nursing, Nursing and Midwifery School, Iran University of Medical Sciences, Tehran, Iran

6 Behavioral Science Integration Kaiser Permanente, The Permanente Medical Group, North Valley, Sacramento, CA, USA 
complications as a result of illness. These actions consist of a series of interdependent behaviors, such as being under the supervision of a specialist, taking medications as directed, exercising, and eating a healthy diet [2-4]. Among persons with a chronic condition, assessment of health behaviors in those with physical disabilities and specifically people with spinal cord injury (SCI), due to its relatively high prevalence, can be considered [5].

In November 2013, the WHO announced that an estimated 250-500 thousand people have a SCI, imposing high costs to persons, families and society $[6,7]$. SCI is a serious medical condition that often causes functional, psychological, and socioeconomic disorders [8-10]. Persons with SCI are required to consider adopting a number of unique health behaviors in addition to the health behaviors practiced by others, including those with chronic illness. Longterm, secondary medical complications are common with SCI and influence the continuum of care for this population. Complications are frequent causes of morbidity and mortality and may lead to increased rates of re-hospitalization, and loss of employment along with decreased quality of life, long term health, dignity, mobility, and independence. Health complications following SCI also are costly in terms of limited health care resources and overall quality of life [11-13].

To reduce the secondary complications and improve the health behaviors in persons with SCI, behavioral interventions may be indicated, but there must be a suitable instrument to measure health behaviors in the SCI population [14, 15]. Pruitt et al. created the Spinal Cord Injury Lifestyle Scale (SCILS) in 1998 as a new measure to assess health behaviors that delay or prevent secondary impairments in individuals with SCI [15]. Subsequently, Bloemen-Vrencken (2007) used the SCILS as a basis for creating a new health behavior questionnaire (HBQ) and several items from the original SCILS instrument were adopted [14]. It is important to have the ability to evaluate the effectiveness of care and interventions such as education and training about health maintenance and prevention for people with SCI. By measuring the health behavior of persons with SCI, it can be determined if current SCI care is meeting these goals [14, 16]. These two standardized questionnaires appear to be quite related to each other and no other instruments to measure health behavior of persons with SCI were found in the literature. Therefore, the aim of this study was to evaluate the reliability and validity of a previously developed lifestyle instrument that measures health behaviors in persons with SCI and a more recent version of this measure as translated into the Persian language. The Persian language version is equivalent to the English version linguistically and semantically. In addition, we measured health behavior outcomes based on the abovementioned questionnaire that can delay or prevent the development of secondary complications associated with SCI.

\section{Method}

The study was cross-sectional and psychometric, performed to evaluate of the validity and reliability of the SCILS and the HBQ in the Persian language. The study protocol was reviewed and approved by Institutional Review Board (IRB) at Tehran University of Medical Sciences.

First, agreement for translating the questionnaires was made with the corresponding author of the original paper describing the development of the SCILS (Pruitt et al. [15]). Next, using standard translation procedures, two authors fluent in English translated the questionnaires into Persian independently (forward translation). Both versions were reviewed by a third translator. The questionnaires were then translated from the Persian language into English again (backward translation), and re-reviewed by two translators who were fluent in English. The Persian translation was deemed to be similar to the original version of the SCILS. Finally, the translators compared the translations, removed and integrated the contradictions, and achieved a Persian version suitable for persons with SCI. The face validity and content validity were approved by 12 professors (11 from the faculty members of the School of Nursing and Midwifery, and one neurosurgeon from Sina Trauma and Surgery Research Center). The face validity was also assessed by ten persons with SCI. All the individuals with SCI and the experts were hesitant and confused on a question that was common between the two questionnaires, and thus, in accordance to the original author (Pruitt et al. [15]), the question was rewritten in an understandable sentence as follows:

"I do some type of pressure relief every $30 \mathrm{~min}$ any time I am in my chair or driving" changed to "I make sure to change my body's position every thirty minutes, whenever I am sitting or using my wheelchair to prevent skin ulcers."

\section{Data collection}

To test the final versions of both questionnaires, 97 persons were included using a consecutive sampling method of all persons with SCI who agreed to participate and met the inclusion criteria, until the numbers of desired participants were recruited. Next, the informed consent form that was consistent with the revised Declaration of Helsinki, was completed by all participants. Participants were selected among those referred to local health centers and the Brain 
and Spinal Cord Injury Research Center. The inclusion criteria were as follows: persons with traumatic SCI (paraplegia and tetraplegia), at least one-year post-injury, able to read and comprehend the Persian language, and age above 18. Participants with cognitive disorders were excluded.

The time interval for test-retest reliability was 14 days in order to be sufficiently short to support the assumption that the persons with SCI remain stable and sufficiently long enough to prevent recall.

After obtaining consent, the questionnaires were completed by interviews with each of the participants. Questionnaires were used to assess concurrent validity (Secondary Impairment Checklist, as well as SCILS and HBQ) and convergent validity (Impact of Event Scale Revised, Brief Symptom Inventory, Beck Depression Inventory, Functional Independence Measure) by interviews also were administered.

\section{SCILS and HBQ}

The SCILS contains five subscales (skin $=7$ items, cardiovascular $=4$ items, genitourinary $=4$ items, neuromuscular $=8$ items, and mental $=2$ items), for a total of 25 items, and the scores are based on a Likert Scale of 0-4 (where $0=$ never, $1=$ rarely, $2=$ sometimes, $3=$ often, and 4 =always). Cronbach's alpha for the subscales were: cardiovascular 0.73 , genitourinary 0.32 , neuromuscular 0.75 , skin 0.86 , and mental 0.31 , and total score 0.81 [15]. The HBQ contains 22 items, and scoring is based on a Likert Scale of 0-3. The questionnaire was derived from the SCILS by Bloemen-Vrencken et al. (with a Cronbach's alpha of 0.8) and reduced to 22 items (some items are new). In both questionnaires, a higher total score indicates greater involvement in healthy behaviors (see Supplementary Material) [14].

\section{Convergent validity}

The following standardized instruments were used for assessing convergent validity of the SCILS and HBQ:

\section{Impact of event scale revised (IES-R)}

The IES-R is a 22 item self-report scale that evaluates mental distress over the last 7 days related to a specific events including SCI. The questionnaire was designed using a 5-point scale, from " $0=$ not at all" to " $4=$ extremely" distressed, containing three subscales of avoidance, intrusion, and over-arousal. In this study, two subscales of Intrusion and Avoidance were used as two measures to evaluate SCILS and HBQ. Cronbach's alpha for avoidance, unwanted thoughts, and over-arousal were $0.87,0.84$, and 0.79 , respectively [17]. Persian version validation in IES-R had good internal consistency with Cronbach's alpha from 0.67 to 0.87 [18].

\section{Brief symptom inventory (BSI)}

The BSI is a 53-item self-report questionnaire used to evaluate the symptoms of psychological distress during the 7 last days. Each BSI item is scored using the 5-point scale, (from " $0=$ not at all" to " $4=$ extremely" distressed). In the present study, two subscales of anxiety (six items) and depression (six items) were used, for which the internal consistency of 0.85 and 0.88 , respectively has been reported [19]. Also, a study of the validity and reliability of the Persian version of the questionnaire reported Cronbach's alpha for the subscales ranging from 0.59 to 0.84 . The ICC measure of test-retest reliability for total score of the BSI was 0.95. [20]

\section{Beck depression inventory (BDI)}

This 21-item inventory evaluates depressive symptoms using a 4-point scale, from " $0=$ symptom absent" to " $3=$ severe symptoms". Internal consistency has been reported as 0.81 for non-mental health samples and 0.86 for mental health samples [21]. A study of validity and reliability of the Persian version of the questionnaire reported the ICC measure of test-retest reliability for total score of the BDI 0.55 , and Cronbach's alpha for the all questions was 0.83 [22].

\section{Functional independence measure (FIM)}

The FIM is a standardized questionnaire containing 18 items designed to assess functional abilities. Items are scored on a scale of $1-7$, with one being totally dependent and seven being totally independent. A total score ranges from 18 to 126. Excellent Cronbach's alpha levels were also found for the English FIM in patients with neurological disorders (0.89-0.98) [23]. A study of the validity and reliability of the Persian version of the questionnaire found Cronbach's alpha for the subscales of the FIM ranging from 0.70 and 0.96 . The ICC measure of test-retest reliability for total score of the FIM was 0.94 .

\section{Concurrent validity}

For concurrent validity assessment, the Secondary Impairment Checklist, as well as the SCILS and HBQ were used. The checklist contains 38 secondary impairments correlated with SCI used by Pruitt et al. In this inventory, participants experiencing secondary impairments right now or during the last 12 months reported "Current", and those who experienced secondary impairments at any time reported 
"Life time". The internal consistency using Cronbach's alpha was 0.8 for current impairments and 0.75 for life time impairments [15, 17, 19, 21, 24, 25].

\section{Statistical methods}

Continuous variables were described using means (SDs) and categorical variables were summarized as a number (percentage). Cronbach's alpha and interclass correlation coefficients (ICC) and Pearson correlation coefficients $(r)$ were used to assess the internal consistency, test-retest (temporal) reliability, and convergent and concurrent validity of the questionnaires, respectively. The statistical software used for the analysis was SPSS 18.

\section{Results}

In this study, 97 persons with SCI participated and completed the questionnaires. The mean (SD) age and duration of SCI were 36.29 (11.49) and 4.36 (4.28) years, respectively. According to the American Spinal Injury Association Impairment Scale (AIS), the participants were divided into two groups of complete or incomplete injury: paraplegia and tetraplegia. Table 1 shows the demographic and baseline characteristics of the participants.

The Cronbach's alphas of the SCILS and HBQ were 0.75 and 0.85 , respectively. The descriptive and internal consistency measures for SCILS subscales are described in Table 2. Test-retest reliability ICC for total scores of the SCILS and HBQ were 0.75 and 0.86 , respectively. The ICCs of 25 questions of the SCILS were between 0.68 and 0.92 , the ICCs of 22 questions of the HBQ were between 0.72 and 0.94 .

The number of current secondary impairments had a weak negative correlation with SCILS $(r=-0.22, P<$ 0.001 ) and the SCILS was strongly correlated with the HBQ $(r=0.65, P<0.001)$ (Table 3).

Total scores of SCILS and the HBQ had a moderate negative correlation and a weak negative correlation with the BDI, respectively $(r=-0.45, P<0.001$, and $r=$ $-0.33, P<0.001)$. The total score of SCILS had a moderate negative correlation with the anxiety dimension $(r=-0.42$, $P<0.001)$ and a moderate negative correlation with the depression dimension $(r=-0.48, P<0.001)$ on the BSI. The total score of HBQ had a significantly weak negative correlation with the depression dimension of the BSI $(r=$ $-0.33, P<0.001$ ). While the total score of SCILS had a weak negative correlation with avoidance $(r=-0.20, P=$ 0.04) of the IES-R scale, the total score of health behaviors had a weak negative correlation with avoidance $(r=-0.32$, $P<0.001)$ and also a weak negative correlation with Intrusion $(r=-0.26, P<0.001)$. Finally, there was a weak positive correlation between the total score of the SCILS
Table 1 Demographic and baseline characteristics of persons with SCI

\begin{tabular}{|c|c|}
\hline Variables & $N(\%)$ \\
\hline \multicolumn{2}{|l|}{ Age } \\
\hline $25 \geq$ & $13(13.4)$ \\
\hline $26-35$ & $47(48.5)$ \\
\hline $36-45$ & $16(16.5)$ \\
\hline $46-55$ & $11(11.3)$ \\
\hline $55 \leq$ & $10(10.3)$ \\
\hline \multicolumn{2}{|l|}{ Sex } \\
\hline Men & $77(79.4)$ \\
\hline Women & $20(20.6)$ \\
\hline \multicolumn{2}{|l|}{ Education } \\
\hline Illiterate & $5(5.2)$ \\
\hline Primary & $7(7.2)$ \\
\hline Guidance & $31(32)$ \\
\hline High school diploma & $38(39.2)$ \\
\hline Collegiate & $16(16.4)$ \\
\hline \multicolumn{2}{|l|}{ Marital status } \\
\hline Single & $33(34)$ \\
\hline Married & $55(56.7)$ \\
\hline Divorced & $9(9.3)$ \\
\hline \multicolumn{2}{|l|}{ SCI duration } \\
\hline $1-5$ & $77(79.4)$ \\
\hline $6-10$ & $10(10.3)$ \\
\hline $11-15$ & $2(2.1)$ \\
\hline $16-20$ & $7(7.2)$ \\
\hline $20 \leq$ & $1(1)$ \\
\hline \multicolumn{2}{|l|}{ Cause of trauma } \\
\hline Transport & $56(57.7)$ \\
\hline Sports and leisure & $6(6.2)$ \\
\hline Fall & $35(36.1)$ \\
\hline \multicolumn{2}{|l|}{ Monthly income } \\
\hline Poor $(<1,000,000$ Toman $)$ & $39(40.2)$ \\
\hline Fair $(1,000,000-3,000,000)$ & $51(52.6)$ \\
\hline Good $(>3,000,000)$ & $7(7.2)$ \\
\hline \multicolumn{2}{|l|}{ Type of injury } \\
\hline Complete & $60(61.9)$ \\
\hline Incomplete & $37(38.1)$ \\
\hline \multicolumn{2}{|l|}{ Level of injury } \\
\hline Paraplegia & $77(79.4)$ \\
\hline Tetraplegia & $20(20.6)$ \\
\hline
\end{tabular}

and the FIM $(r=0.31, P<0.001)$, but not between the total score of the HBQ and the FIM.

\section{Discussion}

Results of this study showed that the SCILS and the revised HBQ are valid and reliable instruments for measuring health 
Table 2 The descriptive and internal consistency measures for SCILS subscales

\begin{tabular}{lllll}
\hline Subscale & Number of items & Mean (SD) & Min-Max & Cronbach's alpha \\
\hline Cardiovascular & 4 & $8.47(4.01)$ & $0-16$ & 0.72 \\
Genitourinary & 4 & $7.98(4.48)$ & $0-16$ & 0.74 \\
Neuromusculoskeletal & 8 & $22.06(6.60)$ & $0-32$ & 0.76 \\
Skin & 7 & $16.21(5.90)$ & $4-28$ & 0.68 \\
Psychosocial & 2 & $5.19(2.15)$ & $0-8$ & 0.73 \\
Total score & 25 & $59.93(14.37)$ & $29-96$ & 0.75 \\
\hline
\end{tabular}

Table 3 Relationship between SCILS total score, Health Behavior total score and validation criteria

\begin{tabular}{lll}
\hline Validation measure & $\begin{array}{l}\text { Pearson r } \\
\text { (SCILS) }\end{array}$ & $\begin{array}{l}\text { Pearson } \mathrm{r} \\
\text { (Health behavior) }\end{array}$ \\
\hline Beck depression inventory (BDI) & $-0.45^{* *}$ & $-0.33^{*}$ \\
Brief symptom inventory (BSI) & & \\
$\quad$ Depression & $-0.48^{* *}$ & $-0.33^{* *}$ \\
$\quad$ Anxiety & $-0.42^{* *}$ & -0.17 \\
Impact of events scale-revised & & \\
(IES-R) & & \\
$\quad$ Intrusion & -0.19 & $-0.26^{* *}$ \\
$\quad$ Avoidance & $-0.20^{*}$ & $-0.32^{* *}$ \\
$\quad$ Functional independence measure & $0.31^{* *}$ & 0.15 \\
(FIM) & & \\
Secondary impairments & & \\
Current & $-0.22^{*}$ & -0.11 \\
Lifetime & -0.12 & -0.12 \\
SCILS & 1 & $0.65^{* *}$ \\
Health behavior & $0.65^{* *}$ & 1
\end{tabular}

* Correlation is significant at the 0.05 level

** Correlation is significant at the 0.01 level

behaviors of persons with SCI. Both questionnaires have good test-retest reliability and internal consistency. The items included in the health behavior measure are more recent. For example, the aspects of genitourinary health behavior are revised from the SCILS, and this change increases usefulness of this questionnaire in persons with incomplete SCI (i.e., those with a sense of urination who typically don't use catheters). The present study population was heterogeneous, including individuals with complete and incomplete SCI. This population difference may be reflected in the performance of more health behaviors than reported the original SCILS study. Pruitt et al. who developed the SCILS had a more homogeneous population $[14,15]$.

Both the SCILS and HBQ should be correlated with each other to demonstrate convergent validity. The SCILS should be negatively correlated with measures of depression and anxiety, distress associated with SCI and functional status. Also, the HBQ is expected to be negatively correlated with measures of depression, and the effect of SCI on Intrusion and Avoidance dimensions. These two questionnaires were not significantly correlated with other structures.

The results of this study were not similar to Pruitt et al. [15] who reported no correlation between depression, anxiety, and effect of SCI and functional independence with the total score of the SCILS. This difference might be due to differences in the sample sizes or non-homogeneity of the population. In the current study, the sample size was larger and a number of participants had incomplete injuries.

In this study, the moderate negative correlation showed that the anxiety and depression are inversely related with the total score on the SCILS and the HBQ in such a way that by increasing the depression score, the health behaviors and SCILS scores are reduced. Tawashy et al. and Bombardier et al. showed that depression is correlated with lifestyle physical activity (personal hygiene, household chores, and work-related activity) [26, 27].

In this study, there was a weak but significant correlation between the FIM and the SCILS. The correlation was not seen with total score of the SCILS. Sandy et al. noted that independence has a strong positive correlation with physical activity and lifestyle, and the loss of independence is associated with reduced quality of life and lifestyle activity of the person with SCI [28].

In order to assess concurrent validity, the correlation between the SCILS and the HBQ was measured, and the secondary impairment checklist was also used, which was only significant for current impairments. This shows that the secondary impairment checklist cannot be a proper criterion for the objective questionnaires of this research, as it was consistent with Pruitt et al. [15]. In the present study current secondary impairments had a weak negative correlation with SCILS in that by increasing the number of current secondary impairments, the SCILS score is reduced. Guilcher et al. showed that secondary complications in persons with SCI are correlated with health behaviors [29]. Jensen also showed that the secondary health conditions in persons with SCI affect different dimensions of lifestyle of these individuals [30]. Thus, health behaviors are known to prevent or delay the onset of secondary impairments in persons with SCI [31]. Also the SCILS and the HBQ were strongly correlated. Tawashy et al. stated that physical activity and healthy lifestyle are related to fewer secondary 
complications [27]. Bloemen-Vrencken also reported that persons with fewer secondary impairments had greater engagement in positive health behaviors, because positive health behavior prevents secondary impairments [14].

\section{Limitations}

One potential limitation of this study was the number of questions asked of the participants. The length of time to complete the questionnaires could create some response burden or fatigue in the participants and possibly affect the precision of their answers.

However, the strengths of this study include (1) using a thoughtful psychometric evaluation of the reliability and validity of two questionnaires used previously to assess health behaviors in persons with SCI and (2) using an adequate sample size.

\section{Conclusion}

According to the results of this psychometric study, the SCILS and the more recent health behavior measure (HBQ) can be used for measuring the health behaviors in persons with SCI in Iran. Both questionnaires can be used as instruments for assessing the education and clinical interventions necessary in persons with SCI. Since health behavior is evaluated with a score from both instruments, the risk of many of the complications can be identified and prevented with early intervention.

\section{Compliance with ethical standards}

Conflict of interest The authors declare that they have no conflict of interest.

\section{References}

1. World Health Organization. The WHO cross-national study of health behavior in school-aged children from 35 countries: findings from 2001-2002. J Sch Health. 2004;74(6):204-6.

2. Sarafino EP, Smith TW. Health psychology: biopsychosocial interactions. 8th edn. Salt Lake City: Wiley, University of Utah; 2014.

3. Schwarzer R. Self-efficacy: thought control of action. New York: Taylor \& Francis; 2014.

4. Schwarzer R, Lippke S, Luszczynska A. Mechanisms of health behavior change in persons with chronic illness or disability: the health action process approach (HAPA). Rehabil Psychol. 2011;56(3):161.

5. Centers for Disease Control Prevention. Prevalence of disabilities and associated health conditions among adults-United States, 1999. MMWR Morb Mortal Wkly Rep. 2001;50(7):120.

6. SCI Prevalence. http://www.who.int/mediacentre/factsheets/fs384/ en/. 2013. Accessed 2 Oct 2014.
7. Wyndaele M, Wyndaele J-J. Incidence, prevalence and epidemiology of spinal cord injury: what learns a worldwide literature survey? Spinal Cord. 2006;44(9):523-9.

8. Myers J, Lee M, Kiratli J. Cardiovascular disease in spinal cord injury: an overview of prevalence, risk, evaluation, and management. Am J Phys Med Rehabil. 2007;86(2):142-52.

9. Charlifue S, Post M, Biering-Sørensen F, Catz A, Dijkers M, Geyh S, et al. International spinal cord injury quality of life basic data set. Spinal Cord. 2012;50(9):672-5.

10. Dietz V, Sinkjaer T. Spastic movement disorder: impaired reflex function and altered muscle mechanics. Lancet Neurol. 2007;6 (8):725-33.

11. Chiodo AE, Scelza WM, Kirshblum SC, Wuermser L-A, Ho CH, Priebe MM. Spinal cord injury medicine. 5. Long-term medical issues and health maintenance. Arch Phys Med Rehabil. 2007;88 (3):S76-S83.

12. Dorsett P, Geraghty T. Health-related outcomes of people with spinal cord injury-a 10 year longitudinal study. Spinal Cord. 2008;46(5):386-91.

13. Hitzig SL, Tonack M, Campbell KA, McGillivray CF, Boschen KA, Richards K, et al. Secondary health complications in an aging Canadian spinal cord injury sample. Am J Phys Med Rehabil. 2008;87(7):545-55.

14. Bloemen-Vrencken J, De Witte L, Post M, Van, den Heuvel W. Health behaviour of persons with spinal cord injury. Spinal Cord. 2007;45(3):243.

15. Pruitt S, Wahlgren D, Epping-Jordan J, Rossi A. Health behavior in persons with spinal cord injury: development nd initial validation of an outcome measure. Spinal Cord 1998;36:724-31.

16. Bloemen-Vrencken J, de Witte L, Post M, Pons C, van Asbeck F, van der Woude L, et al. Comparison of two Dutch follow-up care models for spinal cord-injured patients and their impact on health problems, re-admissions and quality of care. Clin Rehabil. 2007;21(11):997-1006.

17. Creamer M, Bell R, Failla S. Psychometric properties of the impact of event scale-revised. Behav Res Ther. 2003;41 (12):1489-96.

18. Panaghi L, Mogadam JA. Persian version validation in impact of event scale-revised. Tehran Univ Med J TUMS Publ. 2006;64 (3):52-60.

19. Sakakibara BM, Miller WC, Orenczuk SG, Wolfe DL. A systematic review of depression and anxiety measures used with individuals with spinal cord injury. Spinal Cord. 2009;47 (12):841-51.

20. Mamaghani J, Javanmard G. Standardization of brief symptom inventory in counselling and therapeutic situations. J Psychol. 2007;2(8):129-44.

21. Pollard C, Kennedy P. A longitudinal analysis of emotional impact, coping strategies and post-traumatic psychological growth following spinal cord injury: a 10-year review. Br J Health Psychol. 2007;12(3):347-62.

22. Toosi F, Rahimi C, Sajjadi S. Psychometric properties of beck depression inventory-II for high school children in Shiraz City, Iran. Int J School Health 2017;4:e41069.

23. Dodds TA, Martin DP, Stolov WC, Deyo RA. A validation of the functional independence measurement and its performance among rehabilitation inpatients. Arch Phys Med Rehabil. 1993;74 (5):531-6.

24. Anderson K, Aito S, Atkins M, Biering-Sørensen F, Charlifue S, Curt A, et al. Functional recovery measures for spinal cord injury: an evidence-based review for clinical practice and research: report of the national institute on disability and rehabilitation research spinal cord injury measures meeting. J Spinal Cord Med. 2008;31 (2):133-44. 
25. Noreau L, Proulx P, Gagnon L, Drolet M, Laramée M-T. Secondary impairments after spinal cord injury: a population-based study. Am J Phys Med Rehabil. 2000;79(6):526-35.

26. Bombardier CH, Fann JR, Tate DG, Richards JS, Wilson CS, Warren AM, et al. An exploration of modifiable risk factors for depression after spinal cord injury: which factors should we target? Arch Phys Med Rehabil. 2012;93(5):775-81.

27. Tawashy AE, Eng JJ, Lin KH, Tang PF, Hung C. Physical activity is related to lower levels of pain, fatigue and depression in individuals with spinal-cord injury: a correlational study. Spinal Cord. 2008;47(4):301-6.

28. Stevens SL, Caputo JL, Fuller DK, Morgan DW. Physical activity and quality of life in adults with spinal cord injury. J Spinal Cord Med. 2008;31(4):373-8.
29. Guilcher SJ, Craven BC, McColl MA, Lemieux-Charles L, Casciaro T, Jaglal SB. Application of the Andersen's health care utilization framework to secondary complications of spinal cord injury: a scoping review. Disabil Rehabil. 2012;34 (7):531-41.

30. Jensen M, Truitt A, Schomer K, Yorkston K, Baylor C, Molton I. Frequency and age effects of secondary health conditions in individuals with spinal cord injury: a scoping review. Spinal Cord. 2013;51(12):882-92.

31. Vos-Draper TL, Morrow MM. Seating-related pressure injury prevention in spinal cord injury: a review of compensatory technologies to improve in-seat movement behavior. Curr Phys Med Rehabil Rep. 2016;4(4): $320-8$. 